\title{
152 Acute infective sinusitis
}

A Pain is limited to the area overlying the affected sinus.

B Oedema of the overlying tissues is commoner in children.

C Mucopurulent nasal discharge is necessary to make the diagnosis.

D Decongestant nose drops are used to provide 'medical drainage'.

E An antibiotic solution should be instilled into the sinus cavity during antral lavage.

\section{Chronic non-specific sinusitis}
A May be due to an unresolved attack of acute sinusitis.
B Vasomotor rhinitis is the aetiological factor in two-thirds of cases.
C Multiple small abscesses in the thickened mucosa are a recognised pathological feature.
D Bacteriology usually reveals pure cultures of streptococci.
E Postnasal drip is a sine qua non for the diagnosis.

\section{Mixed infective and vasomotor chronic sinusitis}

A Eosinophils and polymorphonucleocytes are found in the discharge.

B The frontal sinus is most commonly affected.

C Polyps are often found and may block the sinus ostia.

D Topical steroid therapy should be avoided because of the danger of uncontrolled infection.

E A radical operation offers a good prospect of permanent cure. 